\title{
Feasibility study for an edge main ion charge exchange recombination spectroscopy system at ASDEX Upgrade
}

\author{
P. Cano-Meaias ${ }^{\mathrm{a}}$. E. Viezzer ${ }^{\mathrm{b}}$. A. Jansen van Vuurenc. M. Cavedon ${ }^{\mathrm{c}}$. D.J. Cruz- \\ Zabala $^{\mathrm{b}}$. R. Dux ${ }^{\mathrm{c}}$, B. Geiger ${ }^{\mathrm{c}}$, M. Garcia-Munoz ${ }^{\mathrm{b}}$, R. Chacartegui $^{\mathrm{a}}$ and the ASDEX \\ Upgrade team ${ }^{c}$ \\ ${ }^{a}$ Dpt. of Energy Engineering, University of Seville, \\ Camino de los Descubrimientos, $s / n$. 41092, Seville \\ ${ }^{b}$ Dpt. of Atomic, Molecular and Nuclear Physics, University of Seville \\ Avda. Reina Mercedes, 41012 Seville, Spain \\ ${ }^{c}$ Max Planck Institute for Plasma Physics \\ Boltzmannstr. 2, 85748 Garching, Germany \\ E-mail: pcano1@us.es
}

ABSTRACT: A feasibility study for a new edge main ion charge exchange recombination spectroscopy (CXRS) system at the ASDEX Upgrade tokamak has been carried out. This diagnostic uses the new edge fast-ion $\mathrm{D}_{\alpha}$ (FIDA) spectrometers and the optical heads of the existing edge CXRS systems. Standard CXRS resolved temperature and rotation profiles rely on the observation of impurity emission lines. The complexity of the photoemission processes and the fact that the measured ions represent the majority bulk plasma ions (here deuterium) make the interpretation of the main ion spectra in comparison more challenging. At the plasma edge, the diagnostic capabilities in terms of temporal and spatial resolution are extremely demanding. The difficulties in the interpretation of the main ion spectra are overcome by comprehensive fitting and forward modelling of the beam halo (which introduces a major challenge) using a collisional radiative model, as described in [S.R. Haskey, JINST 12 (2017) C10013]. In this work, the diagnostic set up and the sensitivity of the main ion spectra to changes in plasma parameters have been characterized. The tools and techniques necessary for a correct interpretation of the spectra are also discussed. In addition to the halo effect, corrections due to atomic physics effects which could affect the splitting and broadening of the spectral $\mathrm{D}_{\alpha}$ line and lead to misinterpretation of the spectra are evaluated. The first measurements of the edge main ion diagnostic are also presented.

KEYWORDS: Plasma diagnostics - charged-particle spectroscopy; Analysis and statistical methods; Data processing method 


\section{Contents}

1. Introduction 1

2. Diagnostic setup 1

3. Sensitivity of the edge main ion $D_{\alpha}$ spectra to plasma parameters 3

4. Interpretation of the edge main ion spectra 5

4.1. Corrections due to spatial distortions related to halo neutrals 5

4.2. Corrections due to cross-section and gyro-motion effects 6

4.3. Corrections due to Zeeman, fine structure and Stark effect 6

$\begin{array}{ll}\text { 5. Conclusions and outlook } & 7\end{array}$

\section{Introduction}

Measurements of the bulk plasma properties are important for understanding fusion plasmas, since they determine plasma performance, confinement and fusion power. While measurements of electron and impurity temperature and density, as well as impurity rotation have high availability, the direct measurement of main ion profiles is more challenging and, for many years, has been rarely done [1]. Given the short thermal equilibration times between main and impurity ions compared to tokamak transport time scales [2], the main ion temperature is traditionally assumed equal to the impurity temperature. Regarding the rotation, differences between main ions and impurities are expected from neoclassical theory [3]. Recent progress on directly measuring main ion properties has been made ([4] and references therein), which has enabled comparison of main ion properties to impurities and neoclassical predictions. In particular, edge main ion charge exchange recombination spectroscopy (CXRS) measurements have been made on DIII-D [5].

In this work, the results of a feasibility study for an edge main ion CXRS system at the ASDEX Upgrade (AUG) tokamak are presented. The first measurements of the diagnostic are discussed. This paper is structured as follows: section 2 gives a description of the CXRS technique, the $\mathrm{D}_{\alpha}$ spectra and the diagnostic set up. The resolution for the new edge main ion CXRS system at AUG is also presented in this section. In section 3, the sensitivity of the $\mathrm{D}_{\alpha}$ spectra to plasma parameters is characterized. In section 4 , the necessary analysis and developed tools for correctly resolving the main ion $\mathrm{D}_{\alpha}$ spectra are discussed. Section 5 gives conclusions and an outlook.

\section{Diagnostic setup}

Charge exchange recombination spectroscopy (CXRS) is a widely extended technique to evaluate the ion temperature and rotation in tokamaks [6]. The technique is based on the observation of spectral lines, typically fully-ionized impurities (because of a relatively simple spectrum) which emit light after a charge exchange reaction with diagnostic neutrals. The intensity and Doppler shift and width of the measured line is related to the apparent density, rotation and temperature of the observed species, respectively. The apparent profiles (obtained directly from fitting the spectral line) might need corrections to resolve the true profiles. This 
need depends on the specific transition, plasma conditions and diagnostic set up (discussed in detail in section 4).

The Balmer $\mathrm{D}_{\alpha}$ emission ( $\mathrm{D} \mathrm{n}=3 \rightarrow 2, \lambda=656.1 \mathrm{~nm}$ ) gives us a substantially more complex spectrum compared to impurities [7]. The edge system at AUG looks at a deuterium neutral beam with a first energy component of $60 \mathrm{keV}$, such that the $\mathrm{CX} \mathrm{D}_{\alpha}$ emission appears together with the beam emission (BES, due to excitation of beam neutrals) $[8,9]$ and the fast-ion $\mathrm{D}_{\alpha}$ (FIDA) [10] contribution, which is typically negligible due to the low fast-ion content at the plasma edge. Figure 1(a) shows a measurement of the active edge main ion spectrum and the fit

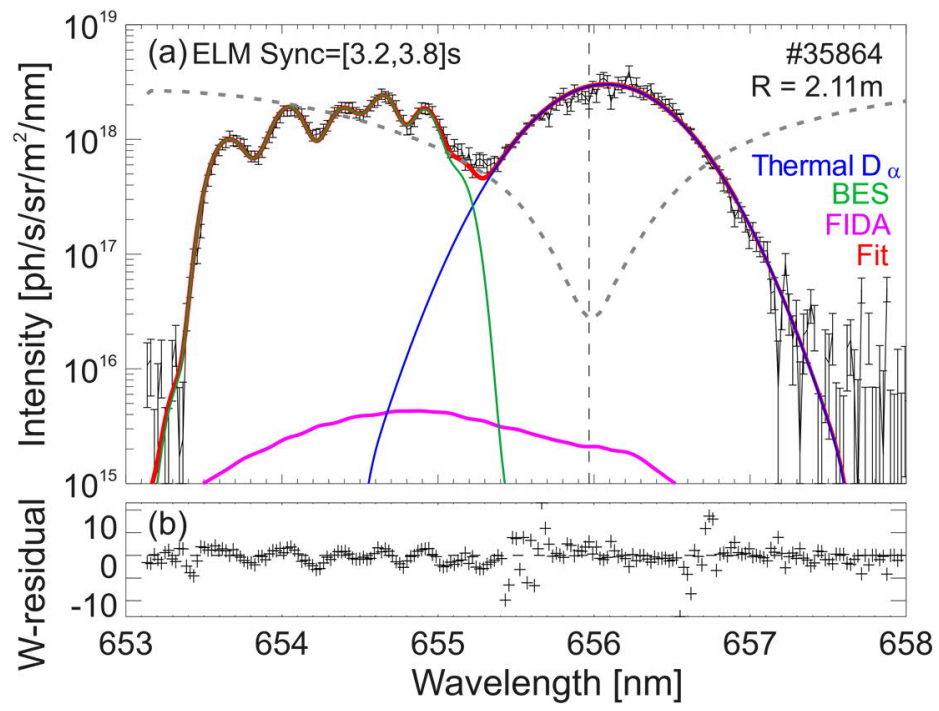

Figure 1. (a) The active edge main ion $\mathrm{D}_{\alpha}$ spectra at AUG. The gray dashed line illustrates the area of influence of the wire which is used to block the cold edge emission (b) Weighted residuals characterize the goodness of the fit.

including the different components. The weighted residuals characterizing the goodness of the fit is shown in (b). Passive background subtraction has been performed by synchronizing passive and active frames with respect to edge localized modes (ELMs) during a time window of $600 \mathrm{~ms}$. This removes more efficiently the cold edge emission and C II impurity lines. The

CX $\mathrm{D}_{\alpha}$ emission (in blue) appears as a single gaussian feature and is due to the superposition of the direct charge exchange (DCX) and halo emission. After the first DCX reaction (between the beam neutral and the thermal plasma), the halo neutrals are born in multiple generations and lead to secondary emission due to electron impact excitation before reionisation. The interpretation of the main ion spectra needs to consider the effect of the halo. This is the biggest challenge in resolving main ion properties and is assessed via forward modelling of the spectra, as will be explained in detail in subsection 4.1.

The edge main ion CXRS at AUG uses the two new edge FIDA spectrometers [11]. The two spectrometers have a dispersion relation of $0.015(0.021) \mathrm{nm} /$ pixel and a time resolution of 1.3 (3.1) ms with a simultaneous imaging of 11 (19) channels. Both use a wire that blocks the intense cold emission and prevents saturation. The grey dotted line in figure 1(a) represents the instrument function of the wire. The spectrometers can be connected to a subset of the 21 toroidal and 19 poloidal lines of sight (LOS) of the standard edge impurity CXRS systems [7,12]. The toroidal and poloidal LOS are separated by $3 \mathrm{~mm}$ and cover $\sim 6 \mathrm{~cm}$ of the pedestal region. Simultaneous measurements of impurities and main ions are possible if the LOS are interleaved. 

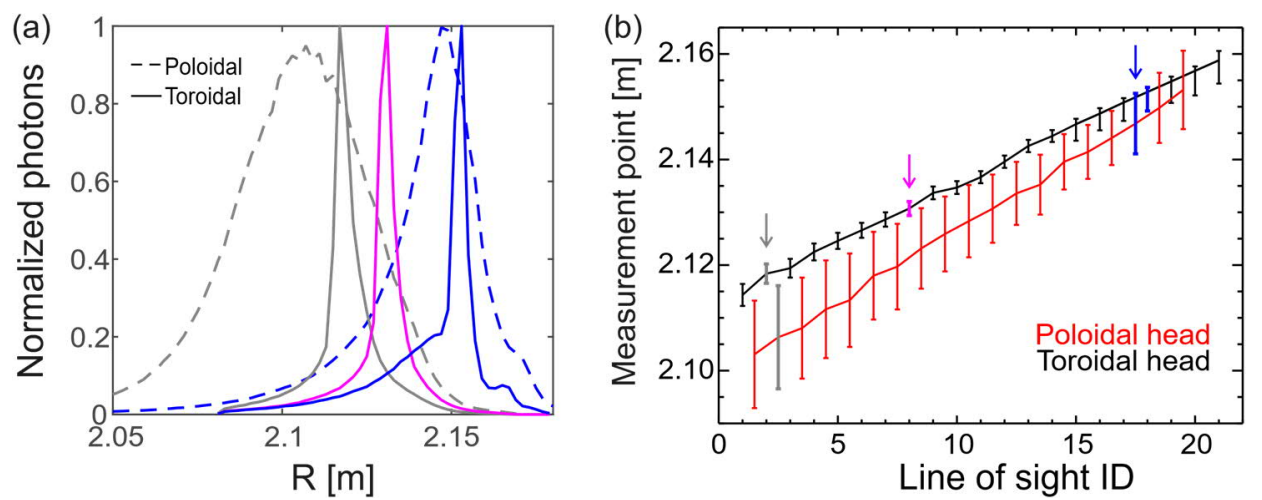

Figure 2. (a) Normalized radial histograms of photons emitted by halo neutrals reaching a specific line of sight. The color code corresponds to the figure in the right. (b) Radial resolution for the edge main ion system using the poloidal and toroidal optical heads of the existing edge CXRS systems. The spatial resolution of the current set up for the thermal $\mathrm{D}_{\alpha}$ emission has been calculated using the FIDASIM code $[13,14]$. FIDASIM is a Monte Carlo code which calculates the neutral density of multiple deuterium populations and their associated photoemission, given kinetic profiles, equilibrium, beam and diagnostic geometry. For the characterization of the spatial resolution of the diagnostic, the origin of photons emitted by halo neutrals arriving to a specific line of sight can be computed [15]. A radial histogram can be built for each LOS, as shown in figure 2(a). The histograms are fitted with Gaussians whose widths are related to the spatial resolution of the diagnostic, as illustrated in figure 2(b). The toroidal and poloidal systems have a spatial resolution of 2 and $10 \mathrm{~mm}$, respectively. The poloidal LOS receive light from a broader region as the poloidal curvature of the plasma is larger compared to the toroidal one. The resolution of the system is minimal just inside the separatrix and increases inwards as the tangency of the LOS to the flux surfaces at the intersection with the beam decreases. These results agree with [12].

Note that for the toroidal LOS outside the separatrix, the blue solid histogram in figure 2(a), a significant amount of light comes from the confined region and a single Gaussian cannot properly describe the radial resolution of the LOS. For this reason, the inner and outer resolution has been characterized for these lines of sight with 2 Gaussians, which gives the asymmetric error bars in figure 2(b). For the poloidal head one Gaussian is enough to describe the radial resolution for LOS measuring inside an outside the separatrix.

\section{Sensitivity of the edge main ion $D_{\alpha}$ spectra to plasma parameters}

In this section, the impact of the electron and ion density and temperature profiles $\left(\mathrm{n}_{\mathrm{e}}, \mathrm{n}_{\mathrm{D}}, \mathrm{T}_{\mathrm{e}}\right.$ and $\mathrm{T}_{\mathrm{D}}$ ) on the main ion spectra will be addressed. To this end, a sensitivity study of the main ion spectra to the plasma parameters has been carried out using the FIDASIM code. In the following, a basic explanation of the contributions of different neutral populations to the spectra is given in terms of their lifetimes. The lifetime of a neutral ( $\left.\tau_{\text {neutral }}\right)$ is inversely proportional to the loss mechanisms of the given neutral population. The lifetimes of the DCX and halo neutrals are given by:

$$
\begin{gathered}
\left(\tau_{\mathrm{DCX}}\right)^{-1}=\mathrm{n}_{\mathrm{e}}\left\langle\sigma_{\text {ion }}^{e} v_{e}\right\rangle+\mathrm{n}_{\mathrm{D}}\left\langle\sigma_{C X} v_{D}\right\rangle \\
\left(\tau_{\text {halo }}\right)^{-1}=\mathrm{n}_{\mathrm{e}}\left\langle\sigma_{\text {ion }}^{e} v_{e}\right\rangle
\end{gathered}
$$

where $\mathrm{n}_{\mathrm{e}}\left(v_{e}\right)$ and $\mathrm{n}_{\mathrm{D}}\left(v_{D}\right)$ are the electron and ion density (collision velocity) and $\langle\sigma v\rangle$ is the reaction rate. Here, $\sigma_{C X}$ and $\sigma_{i o n}^{e}$ are the cross-sections due charge exchange and electron impact ionization. In steady state, from the ratio between the production and loss of halo neutrals, the DCX/halo brightness ratio can be related to the DCX/halo density ratio. It can be expressed as:

$$
\mathrm{n}_{\mathrm{DCX}} / n_{\text {halo }}=\mathrm{n}_{\mathrm{e}}\left\langle\sigma_{\text {ion }}^{e} v_{e}\right\rangle / \mathrm{n}_{\mathrm{D}}\left\langle\sigma_{C X} v_{D}\right\rangle
$$


Increasing the electron density $\left(\mathrm{n}_{\mathrm{e}}\right)$ at the plasma edge results in a decrease of the halo and DCX lifetime, such that the DCX and halo emission are reduced. As the halo decreases more rapidly compared to the DCX, the DCX/halo ratio increases when increasing $\mathrm{n}_{\mathrm{e}}$. The ion temperature $\left(T_{D}\right.$ ) has a strong impact on the spectra (due to $v_{D} \propto \sqrt{T_{D}}$ ), such that increasing $T_{D}$ decreases the DCX emission. For typical $T_{D}$ values at the plasma edge, the DCX contribution is dominant. The impact of the electron temperature is small, as the ionization rate due to collisions with electrons stays rather constant for the typical electron temperatures at the steep
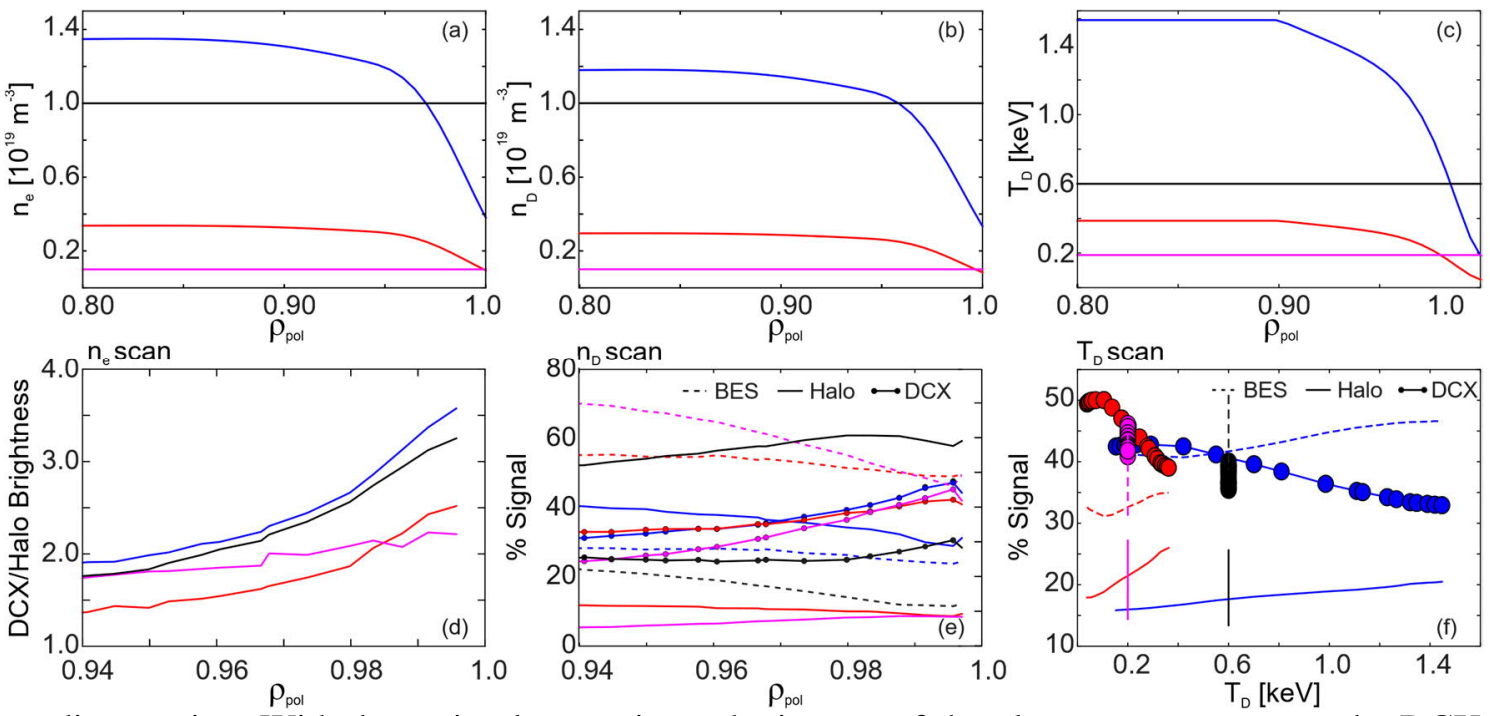

gradient region. With these simple equations, the impact of the plasma parameters on the DCX and halo contributions to the spectra can be understood.

The analysis complicates when one considers multiple generations of halo neutrals and at regions with strong gradients, such as the plasma edge. The FIDASIM code provides an excellent tool for a sensitivity study of the main ion spectra to the parameters. A scan in the $\mathrm{n}_{e}, \mathrm{n}_{\mathrm{D}}, \mathrm{T}_{\mathrm{e}}$ and $\mathrm{T}_{\mathrm{D}}$ profiles has been performed individually, such that their impact on the $\mathrm{D}_{\alpha}$ spectra is isolated. The contribution of the BES, halo and DCX to the total signal or the ratio DCX/halo brightness are compared for each parameter scan. Figures 3(a-c) show the inputs to FIDASIM for each parameter scan and figures 3(d-f) illustrate

Figure 3. Input profiles were varied individually for each scan (a-c). The DCX/halo brightness is shown in (d) while (e-f) show the contribution of each feature in percent. Note the different ranges in the X-axis.

the individual sensitivity to changes in the parameters, e.g. the impact of changes in electron density in figure 3(a) are shown in figure 3(d). When the electron density $\left(n_{e}\right)$ increases at the plasma edge, the DCX and halo emission decrease. As the halo decreases more rapidly compared to the DCX, the DCX/halo ratio increases when increasing $\mathrm{n}_{\mathrm{e}}$. Since the measurements are line-integrated, regions with different gradients are probed. We can see that gradients complicate the dependence by comparing the two low $n_{e}$ cases. Figures $3(b, e)$ show that higher main ion density $\left(n_{D}\right)$ increases the halo contribution, while the DCX/halo ratio decreases and can reach values below 1. This implies that the impact of $n_{e}$ on the spectra is complex, as $n_{D}$ is determined by quasi-neutrality. In this sense, the effects of both electron and ion densities can decouple by means of the effective charge. For the electron temperature, the dependence is small and not shown here. The ion temperature has a strong impact, 
see figure 3(c,f). Higher $\mathrm{T}_{\mathrm{D}}$ means lower DCX and higher BES relative signal, while the halo relative signal is rather constant. There are strong variations in the DCX/halo ratio for typical $T_{D}$ values at the plasma edge, e.g. from $200-400 \mathrm{eV}$ the ratio changes a factor of 2, see figure 3(f). For the $T_{D}$ values considered in the scan, the DCX contribution is dominant over the halo, in agreement with [16].

\section{Interpretation of the edge main ion spectra}

Multiple effects can lead to spectral line broadening and splitting such that the interpretation of the main ion spectra (together with passive background subtraction and comprehensive fitting) relies on correcting for the atomic physic processes which can significantly affect the spectra. In this section, we will see that the $\mathrm{D}_{\alpha}$ spectra need to be corrected for the halo effect. In addition, the cross-section, Zeeman and fine structure corrections are non-negligible for the edge main ion spectra.

\subsection{Corrections due to spatial distortions related to halo neutrals}

In order to correct for the halo and its line integration effects the collisional radiative model implemented in FIDASIM is used. The different contributions to the $\mathrm{D}_{\alpha}$ spectra can be modelled with FIDASIM, such that the predicted spectra for a specific input can be compared with the experimentally measured spectra. FIDASIM has been previously used to interpret the core $\mathrm{D}_{\alpha}$ spectra, by the definition of correction factors [8] and the construction of look-up tables [16]. At the plasma edge, these approaches are insufficient: the LOS sample regions with steep gradients, thus collecting light emitted by hotter (pedestal top) and colder (towards the separatrix) neutrals. The adopted approach for resolving the halo effects at the plasma edge is by running FIDASIM iteratively and comparing the profiles inferred from the synthetic spectra to the experimental profiles [17]. The following steps, illustrated in the workflow in figure 4, are
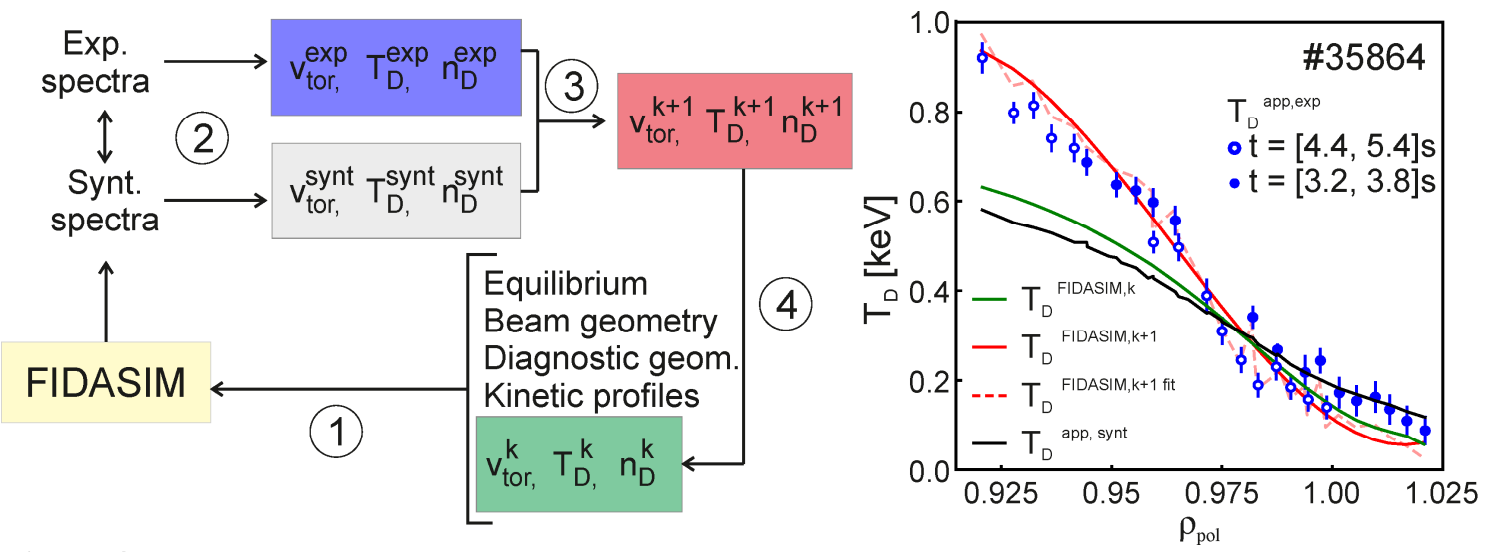

Figure 4. (Left) Workflow to resolve main ion properties. (Right) Color-coded main ion temperature profiles at each step are given as an example.

taken for forward modelling the spectra:

(1) FIDASIM is run for a set of input profiles (an initial guess for the main ion profiles is given).

(2) The synthetic (FIDASIM output) and experimentally measured spectra are fitted, giving apparent synthetic and experimental profiles, respectively. A check for convergency is done. (3) The synthetic and experimental profiles are compared, providing a direction for modifying the next set of input profiles.

(4) The next FIDASIM run is initiated with a modified set of input profiles. 
After each simulation, the experimental and synthetic spectra are compared and convergency is reached when agreement is found within the experimental uncertainty. At this point, also the experimental and synthetic apparent profiles match and the true underlying deuterium properties are found.

In the right plot in figure 4, first apparent edge main ion temperature measurements are shown in blue. A plasma radial sweep of $10 \mathrm{~mm}$ was performed during the experiment to increase the radial coverage of the edge diagnostic. The different markers (open and coloured circles) refer to two different time windows of constant outer radial position. The frames were ELM synchronized as described in section 2. The measurements derived from poor fits have been discarded. Here, the impurity rotation measurements are given as input to FIDASIM. Note that while the iterative process should be applied to resolve the main ion profiles for each time point separately, the radial sweep technique could help to constrain the initial apparent profiles.

\subsection{Corrections due to cross-section and gyro-motion effects}

The cross-section distortion is due to the energy dependence of the cross-section, such that ions moving towards and away from the beam will have a different probability of undergoing a CX reaction [18]. This effect depends on the geometry of the LOS with respect to the beam and leads to a distortion of the spectral line which is not associated to the true ion temperature and velocity, due to an enhancement of the intensity of the spectral line on one side. The crosssection corrections for impurities have been calculated at AUG and are found to be negligible for the edge and core systems [7, 19]. For the main ions, the cross-section effect leads to an error up to $10 \mathrm{eV}$ for temperature and up to $5 \mathrm{~km} / \mathrm{s}$ for rotation. The correction for rotation is rather independent of the observed rotation, such that the correction can be important at the edge, since small toroidal rotation values could be expected in the pedestal region. This correction is automatically included when forward modelling the $\mathrm{D}_{\alpha}$ spectra in FIDASIM, since FIDASIM calculates the reaction rates considering the velocity vector of the beam or halo markers with a thermal distribution of main ions, when calculating the photoemission. The gyromotion correction depends on the lifetime of the excited species and its angular frequency [20]. As opposed to impurities, the $\mathrm{D}_{\alpha}$ emission would not be affected by the gyromotion effect, since deuterium radiates as a neutral atom and would not be affected by the magnetic field (i.e. $\omega=0$, such that the effect vanishes).

\subsection{Corrections due to Zeeman, fine structure and Stark effect}

The Zeeman and Stark effects lead to the splitting of spectral lines in the presence of a magnetic or electric field, respectively. Each Zeeman component is a Gaussian line itself with a Doppler width representing the true ion temperature. When these lines add up, a line shape that is well described by a Gaussian is produced. However, the width of the resulting Gaussian gives an apparent ion temperature which overestimates the real ion temperature. An estimation of the maximal symmetrical splitting [21], gives a splitting of $0.0404 \mathrm{~nm}$ for $\mathrm{D}(\mathrm{n}=3 \rightarrow 2)$, compared to $0.0229 \mathrm{~nm}$ for $\mathrm{B}^{5+}(\mathrm{n}=7 \rightarrow 6)$ at $\mathrm{B}=2 \mathrm{~T}$. The full Zeeman pattern for the $\mathrm{D}(\mathrm{n}=3 \rightarrow 2)$ consists of 54 lines [22]. At AUG, all CXRS $\mathrm{T}_{\mathrm{i}}$ measurements are routinely corrected for Zeeman broadening by using correction factors [23], which include Zeeman as well as fine structure effects $[7,19]$. The calculation of the correction factors assumes fully mixed populations of the $l$ states. This has been checked to be a reasonable assumption using the empirical formula in [24], which gives a critical density above which a given transition is fully mixed. The critical density for the main ions $\left(\mathrm{n}_{\text {crit, }} \mathrm{D}=1.04 \cdot 10^{17} \mathrm{~m}^{-3}\right)$ is two orders of magnitude smaller than typical densities at the plasma edge of AUG $\left(\sim \mathrm{n}_{\mathrm{e}}=1 \cdot 10^{19} \mathrm{~m}^{-3}\right)$. 
These correction factors have been compared with [25], which includes the Zeeman and Stark effect. The Stark effect is negligible at the plasma edge of AUG, while the fine structure results in broader and asymmetric lines. Thus, the correction for Zeeman and fine structure effects is included for the main ions, while the Stark effect correction is not. The correction increases with decreasing apparent temperature and views parallel to the magnetic field ( $\sigma$-lines become dominant) and is typically under $10 \mathrm{eV}$ at the plasma edge. The full Zeeman pattern is convolved with the instrument function and included in the fit to the experimental data. Likewise, since the Zeeman splitting is not included in FIDASIM, the synthetic spectrum is convolved with both the Zeeman pattern and the instrument function for a comparison with the experimental spectrum (step 2 in the workflow in figure 4).

\section{Conclusions and outlook}

The results of a feasibility study for an edge main ion CXRS system at AUG have been presented. The spatial resolution of the diagnostic has been characterized and, together with the temporal resolution of the spectrometers, will allow us to resolve the main ion profiles in the steep gradient region. The data analysis tools necessary for a correct interpretation of the main ion spectra (due to the halo and atomic physics effects which might lead to a misinterpretation of the main ion properties if not included) have been completed and first measurements have been obtained (figure 4). Future work focuses on the interpretation of the main ion spectra by means of the workflow presented in figure 4. This will allow us to resolve the first edge main ion profiles at AUG and give us direct measurements of the main ion properties, which will be compared to impurity properties and neoclassical predictions.

\section{Acknowledgments}

This work has received funding from the Spanish Ministry of Science, Innovation and Universities (grant FPU17/06273), the H2020 Marie-Sklodowska Curie programme (Grant No. 708257), the Spanish Ministry of Economy and Competitiveness (Grant No. FJCI-201422139). This work has been carried out within the framework of the EUROfusion Consortium and has received funding from the Euratom research and training programme 2014-2018 and 2019-2020 under grant agreement No 633053. The views and opinions expressed herein do not necessarily reflect those of the European Commission.

\section{References}

[1] M G von Hellermann et al., Complex spectra in Fusion Plasmas Phys. Scr. 200519 (2005)

[2] E. Viezzer et al., High-accuracy characterization of the edge radial electric field at ASDEX Upgrade Nucl. Fusion 53053005 (2013)

[3] F. L. Hinton and R. D. Hazeltine. Theory of plasma transport in toroidal confinement systems Rev. Mod. Physics, 48 239, 1976.

[4] S.R. Haskey et al., Main ion and impurity edge profile evolution across the L-to H-mode transition on DIII-D Plasma Phys. Control. Fusion 60105001 (2018)

[5] S.R. Haskey et al., Active spectroscopy measurements of the deuterium temperature, rotation, and density from the core to scrape off layer on the DIII-D tokamak Rev. Sci. Instrum 89, 10D110 (2018)

[6] J. Fonck et al. Determination of plasma-ion velocity distribution via charge-exchange recombination spectroscopy Phys. Rev. A, 29(6) (1984)

[7] E. Viezzer et al., High-resolution charge exchange measurements at ASDEX Upgrade Rev. Sci. Instrum. 83, 103501 (2012)

[8] B. Geiger et al., Measurement and interpretation of active Balmer alpha spectra at ASDEX Upgrade, $42^{\text {nd }}$ EPS Conference on Plasma Physics, P1.116 (2015) 
[9] R. Dux et al., A forward model for beam emission spectroscopy at ASDEX Upgrade, $42^{\text {nd }}$ EPS Conference on Plasma Physics, P1.121 (2015)

[10] W. W. Heidbrink et al., Hydrogenic fast-ion diagnostic using Balmer-alpha light Plasma Phys. Control. Fusion 581855 (2004)

[11] A. Jansen van Vuuren, An edge fast-ion D-alpha system installed at ASDEX Upgrade, Rev. Sci. Instrum. 90 (2019) 103501.

[12] M. Cavedon et al., A fast edge charge exchange recombination spectroscopy system at the ASDEX Upgrade tokamak Rev. Sci. Instru400m. 88043103 (2017)

[13] W.W. Heidbrink et al., A Code that Simulates Fast-Ion D $\alpha$ and Neutral Particle Measurements Commun. Comput. Phys. 10716 (2011)

[14] B. Geiger, Fast-ion transport studies using FIDA spectroscopy at the ASDEX Upgrade tokamak, PhD thesis, LMU München (2013)

[15] M. Weiland et al., Enhancement of the FIDA diagnostic at ASDEX Upgrade for velocity space tomography Plasma Phys. Control. Fusion 58025012 (2016)

[16] B. Grierson et al., Active spectroscopic measurements of the bulk deuterium properties in the DIII-D tokamak Rev. Sci. Instrum 83, 10 D529 (2012)

[17] S.R. Haskey et al., Deuterium charge exchange recombination spectroscopy from the top of the pedestal to the scrape off layer in H-mode plasmas 2017 JINST $12 \mathrm{C} 10013$

[18] M von Hellermann et al., Analytical approximation of cross-section effects on charge exchange spectra observed in hot fusion plasmas Plasma Phys. Control. Fusion 3771 (1995)

[19] R.McDermott et al., Extensions to the charge exchange recombination spectroscopy diagnostic suite at ASDEX Upgrade Rev. Sci. Instrum. 8873508 (2017)

[20] R. E. Bell and E. J. Synakowski New understanding of poloidal rotation measurements in a Tokamak plasma AIP Conference Proceedings 39547 (2000)

[21] C Isler An overview of charge-exchange spectroscopy as a plasma diagnostic Plasma Phys. Control. Fusion 36171 (1994)

[22] A. Blom and C. Jupén Parametrization of the Zeeman effect for hydrogen-like spectra in hightemperature plasmas Plasma Phys. Control. Fusion 441229 (2002)

[23] J. D. Hey et al., Doppler Broadening and Magnetic Field Effects on Some Ion Impurity Spectra Emitted in the Boundary Layer of a Tokamak Plasma Contrib. Plasma Phys. 34 6, 725-747(1994)

[24] D. H. Sampson. On statistical equilibrium among the sublevels of hydrogenic atoms and ions J. Phys. B: At. Mol. Phys., 10(4):749 (1977)

[25] J. Rosato, Y. Marandet, R. Stamm. A new table of Balmer line shapes for the diagnostic of magnetic fusion plasmas Journal of Quantitative Spectroscopy \& Radiative Transfer 187 333-337 (2017) 